\title{
Genomic basis of antibiotic resistance in Vibrio parahaemolyticus strain JPA1
}

\section{Felipe Hernandes Coutinho', Diogo Antonio Tschoeke', Maysa Mandetta Clementino², Cristiane Carneiro Thompson', Fabiano Lopes Thompson ${ }^{1,3} /+$}

${ }^{1}$ Universidade Federal do Rio de Janeiro, Instituto de Biologia, Rio de Janeiro, RJ, Brasil

${ }^{2}$ Fundação Oswaldo Cruz-Fiocruz, Instituto Nacional de Controle de Qualidade Sanitária, Rio de Janeiro, RJ, Brasil

${ }^{3}$ Universidade Federal do Rio de Janeiro, Instituto Alberto Luiz Coimbra de Pós-Graduação e Pesquisa de Engenharia,

Laboratório de Sistemas Avançados de Gestão da Produção, Rio de Janeiro, RJ, Brasil

A multi-resistant strain of Vibrio parahaemolyticus was isolated from a tropical estuary in Rio de Janeiro, Brazil. Genome sequencing was conducted to establish the molecular basis of antibiotic resistance in this organism. The genetic content of this strain revealed it to be a non-virulent lineage that nevertheless possesses several antibiotic resistance determinants.

Key words: Vibrio parahaemolyticus - antibiotic resistance - urban lagoon - multi-drug resistance

Virulent strains of Vibrio parahaemolyticus are responsible for several global outbreaks of gastroenteritis caused by the ingestion of contaminated seafood. ${ }^{(1,2)}$ This organism is typically found in warm aquatic environments and is often associated with invertebrates, either adopting a virulent or non-virulent lifestyle. ${ }^{(3,4,5,6)}$ Although several antibiotic-resistant strains of $V$. parahaemolyticus have been reported, ${ }^{(4,6,7)}$ little has been done to elucidate the genetic basis of resistance among the environmental lineages. To tackle this issue, we isolated a multi-resistant strain of $V$. parahaemolyticus, hereby named strain JPA1, from the waters of the Jacarepaguá lagoon system situated in the city of Rio de Janeiro, Brazil. The local population often comes in contact with the waters at this site either directly for recreational purposes or indirectly through the consumption of seafood retrieved from the lagoon system. Despite this, the Jacarepaguá lagoons receive massive amounts of untreated sewage daily; a factor that contributes to the high abundance and diversity of antibiotic-resistant bacteria in this habitat..$^{(8,9)}$ Therefore, understanding the diversity of the antibiotic-resistant bacteria dwelling in the Jacarepaguá lagoons and their molecular mechanisms of resistance can provide insights into the potential risks that these organisms pose to the local population and elucidate how resistance can spread among aquatic bacteria in this habitat.

doi: 10.1590/0074-02760190053

Financial support: CAPES, CNPq, FAPERJ.

+ Corresponding author: fabianothompson1@gmail.com

(D) https://orcid.org/0000-0002-7562-1683

Received 6 February 2019

Accepted 25 March 2019
The antibiotic susceptibility profile of strain JPA1 was determined by measuring the minimum inhibitory concentration (MIC) of 16 drugs against JPA1. This organism was resistant to eight out of the 16 tested antibiotics (Table I). JPA1 showed resistance or intermediate resistance to all the tested beta-lactams, with the exception of ceftriaxone. However, it tested susceptible to all aminoglycosides, tigecycline, and ciprofloxacin.

DNA was prepared for sequencing using the Nextera XT DNA library prep kit following manufacturer's recommendations. Genome sequencing was conducted using the Illumina MiSeq platform that yielded 1,461,209 reads (average length $=250 \mathrm{bp}$ and average Phred score $=37$ ). Reads were subjected to a hybrid assembly using A5(10) and SPAdes. ${ }^{(1)}$ The $5.1 \mathrm{Mbp}$ draft genome of the V. parahaemolyticus strain JPA1 was assembled into 793 scaffolds $(\mathrm{N} 50=17,960 \mathrm{bp})$ and displayed a $\mathrm{G}+\mathrm{C}$ content of $45.1 \%$. Gene prediction was carried out using Prokka, ${ }^{(12)}$ and the predicted proteins were annotated using Diamond $^{(13)}$ for best-hit classification against the NCBI nr database. The assembled genome was deposited in the European Nucleotide Archive under project PRJEB31105.

Clinical strains of $V$. parahaemolyticus often carry genes that encode a type three secretion system (T3SS) for a thermostable direct haemolysin (TDH) and/or TDH-related haemolysin..$^{(1,14)}$ However, neither were detected in the genome of $V$. parahaemolyticus JPA1, suggesting it to be non-virulent to humans. Yet the JPA1 genome encoded genes that were involved in resistance against several classes of antibiotics (Table II). We did not detect these genes in association with any mobile genetic elements, which shows that these are intrinsic resistance mechanisms. Genes coding for three main resistance mechanisms were identified: multi-drug efflux pumps, antibiotic inactivation, and target protection. Efflux pumps confer resistance by pumping antibiotics and other drugs out of the bacterial cytoplasm. Among the efflux pumps identified in the JPA1 genome, those associated with resistance to aminoglycosides, beta-lactams, fluoroquinolones, macrolides, streptogramin, and tet- 
racycline were found. JPA1 also possessed genes for the assembly of the AcrEF-TolC complex, which is a multi-drug efflux pump capable of removing a broad array of drugs from the bacterial cytoplasm. Genes for the MacAB-TolC complex, which grants resistance to macrolides, were also detected. Other cases of efflux pumps conferring resistance are as follows: novA, which encodes an $\mathrm{ABC}$ type III transporter that confers resistance to novobiocin; vgaE, which confers resistance to streptogramin; tet 34 and tet 35 , both of which confer resistance to tetracyclines; and sav1866, which encodes a non-specific multi-drug transporter.

Three genes encoding proteins capable of antibiotic inactivation were also detected: $A P H\left(3^{3}\right)$ ) $I b$ encodes an aminoglycoside-3'-phosphotransferase capable of inactivating aminoglycosides through phosphorylation, $C A R B$ - 18 encodes a $\beta$-lactamase, and catB8 encodes a chloramphenicol acetyltransferase that inactivates amphenicols. Target protection proteins work by impairing the contact between antibiotics and their targets. Gene $d f r A 3$ encodes an alternative dihydrofolate reductase that is less sensitive to the action of trimethoprim. Genes otrA, tetW, and tet32 encode peptides that perform non-covalent modifications to bacterial ribosomes, rendering them resistant to tetracyclines. Furthermore, $q n r C$ and $q n r V C 5$ also contribute to target protection mechanisms that confer resistance to quinolones.

Upon infection, human pathogens are often challenged by antibiotic therapy, which favours strains that possess antibiotic resistance determinants. Many potentially pathogenic bacteria possibly have a free-living lifestyle that includes surviving in soils, water bodies, and associated to non-human hosts. JPA1's genetic con- tent indicates that it is non-pathogenic to humans, despite possessing a broad array of antibiotic resistance genes. Antibiotic resistance genes precede the advent of antibiotic therapy, ${ }^{(15,16)}$ which indicates that these genes may play a different role in bacterial physiology under non-clinical settings. ${ }^{(17)}$ This particularly explains the prevalence of antibiotic resistance genes in the JPA1 genome.

Thus, we conclude that the JPA1 genome has a broad array of antibiotic resistance genes that confer upon it a multi-resistant phenotype. Horizontal gene transfer has been implicated as a mechanism for the acquisition of virulence $^{(18)}$ and antibiotic resistance ${ }^{(19)}$ genes in $V \cdot p a$ rahaemolyticus. In the environment, horizontal gene transfer is often mediated by plasmids and other mobile genetic elements. However, the resistance mechanisms identified in the JPA1 genome were not found to be associated with such elements. Nevertheless, horizontal gene transfer can also take place through the direct uptake of exogenous DNA or via phage-mediated transduction. In the advent that antibiotic resistance genes from JPA1 are mobilised to other bacteria through the aforementioned mechanisms, this strain could play a role in the spread of antibiotic resistance genes in aquatic ecosystems. ${ }^{(6)}$ This is of particular importance considering that JPA1 shares its habitat with many potentially pathogenic organisms that are medically relevant such as Vibrio cholerae, Shigella spp., and Pseudomonas aeruginosa. ${ }^{(9)}$

\section{AUTHORS' CONTRIBUTION}

FHC, MMC, and FLT designed the experiments; FHC and DAT performed the experiments and analysed the data; FHC, DAT, MMC, CC and FLT wrote the manuscript. The authors declare no conflicts of interest.

\section{TABLE I}

Antibiotic susceptibility profile of Vibrio parahaemolyticus strain JPA1

\begin{tabular}{lccc}
\hline Antibiotic & Class & MIC $(\mu \mathrm{g} / \mathrm{mL})$ & Phenotype \\
\hline Ceftriaxone & Beta-Lactam & 8 & Susceptible \\
Meropenem & Aminoglycoside & 4 & Susceptible \\
Amikacin & Aminoglycoside & 16 & Susceptible \\
Gentamicin & Aminoglycoside & 2 & Susceptible \\
Ciprofloxacin & Ciprofloxacin & $<=0.25$ & Susceptible \\
Tigecycline & Glycycycline & $<=0.5$ & Susceptible \\
Piperacillin/Tazobactam & Beta-Lactam & 64 & Intermediate \\
Imipenem & Beta-Lactam & 8 & Intermediate \\
Ampicillin & Beta-Lactam & $>=32$ & Resistant \\
Ampicillin/Sulbactam & Beta-Lactam & $>=32$ & Resistant \\
Cefuroxime & Beta-Lactam & $>=64$ & Resistant \\
Cefuroxime Axetil & Beta-Lactam & $>=64$ & Resistant \\
Cefoxitin & Beta-Lactam & $>=64$ & Resistant \\
Ceftazidime & Beta-Lactam & 32 & Resistant \\
Cefepime & Beta-Lactam & $>=64$ & Resistant \\
Colistin & Polymyxin & 4 & Resistant \\
\hline
\end{tabular}

MIC: minimum inhibitory concentration. 


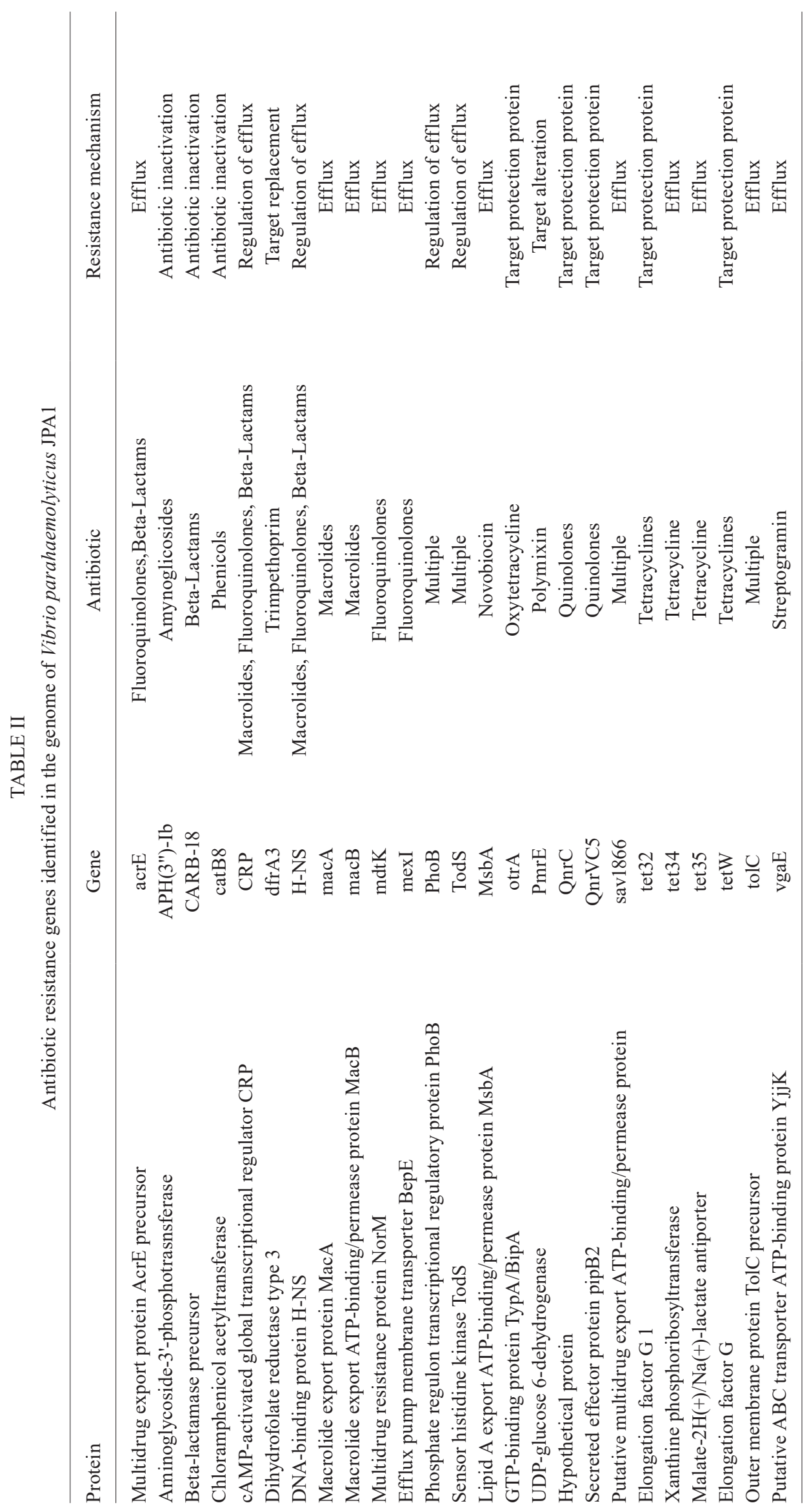




\section{REFERENCES}

1. Su YC, Liu C. Vibrio parahaemolyticus: a concern of seafood safety. Food Microbiol. 2007; 24(6): 549-58.

2. Thompson F, Iida T, Swings J. Biodiversity of vibrios. Microbiol Mol Biol Rev. 2004; 68(3): 403-31.

3. Kondo H, Tinwongger S, Proespraiwong $\mathrm{P}$, Mavichak R, Unajak $\mathrm{S}$, Nozaki R. Draft genome sequences of six strains of Vibrio parahaemolyticus isolated from early mortality syndrome / acute hepatopancreatic necrosis disease shrimp in Thailand. Genome Announc. 2014; 2(2): 1-2.

4. Bhattacharya M, Choudhury P, Kumar R. Antibiotic- and metal-resistant strains of Vibrio parahaemolyticus isolated from shrimps. Microb Drug Resist. 2000; 6(2): 171-2.

5. Kongrueng J, Yingkajorn M, Bunpa S, Sermwittayawong N, Singkhamanan K, Vuddhakul V. Characterization of Vibrio parahaemolyticus causing acute hepatopancreatic necrosis disease in southern Thailand. J Fish Dis. 2014; 38(11): 957-66.

6. Baker-Austin C, McArthur JV, Tuckfield RC, Najarro M, Lindell $\mathrm{AH}$, Gooch J, et al. Antibiotic resistance in the shellfish pathogen Vibrio parahaemolyticus isolated from the coastal water and sediment of Georgia and South Carolina, USA. J Food Prot. 2008; 71(12): 2552-8.

7. Han F, Walker RD, Janes ME, Prinyawiwatkul W, Ge B. Antimicrobial susceptibilities of Vibrio parahaemolyticus and Vibrio vulnificus isolates from Louisiana Gulf and retail raw oysters. Appl Environ Microbiol. 2007; 73(21): 7096-8.

8. Coutinho FH, Silveira CB, Pinto LH, Salloto GRB, Cardoso AM, Martins OB, et al. Antibiotic resistance is widespread in urban aquatic environments of Rio de Janeiro, Brazil. Microb Ecol. 2014; 68(3): 441-52.

9. Salloto GRB, Cardoso AM, Coutinho FH, Pinto LH, Vieira RP, Chaia $\mathrm{C}$, et al. Pollution impacts on bacterioplankton diversity in a tropical urban coastal lagoon system. PLoS One. 2012; 7(11): e51175.
10. Tritt A, Eisen JA, Facciotti MT, Darling AE. An integrated pipeline for de novo assembly of microbial genomes. PLoS One. 2012; 7(9): e42304.

11. Bankevich A, Nurk S, Antipov D, Gurevich A, Dvorkin M, Kulikov AS, et al. SPAdes: a new genome assembly algorithm and its applications to single-cell sequencing. J Comput Biol. 2012; 19(5): 455-477.

12. Seemann T. Prokka: rapid prokaryotic genome annotation. Bioinformatics. 2014; 30(14): 2068-9.

13. Buchfink B, Xie C, Huson DH. Fast and sensitive protein alignment using DIAMOND. Nat Methods. 2015; 12(1): 59-60.

14. Okada N, Iida T, Park KS, Goto N, Yasunaga T, Hiyoshi H, et al. Identification and characterization of a novel type III secretion system in trh-positive Vibrio parahaemolyticus strain TH3996 reveal genetic lineage and diversity of pathogenic machinery beyond the species level. Infect Immun. 2009; 77(2): 904-13.

15. D'Costa VM, King CE, Kalan L, Morar M, Sung WW, Schwarz C, et al. Antibiotic resistance is ancient. Nature. 2011; 477(7365): 457-61.

16. Forsberg KJ, Reyes A, Wang B, Selleck EM, Sommer MO, Dantas G. The shared antibiotic resistome of soil bacteria and human pathogens. Science. 2012; 337(6098): 1107-11.

17. Martínez JL. Antibiotics and antibiotic resistance genes in natural environments. Science. 2008; 321(5887): 365-7.

18. Chen Y, Stine OC, Badger JH, Gil AI, Nair GB, Nishibuchi M, et al. Comparative genomic analysis of Vibrio parahaemolyticus: serotype conversion and virulence. BMC Genomics. 2011; 12(1): 294-306

19. Ceccarelli D, Salvia AM, Sami J, Cappuccinelli P, Colombo MM. New cluster of plasmid-located class 1 integrons in Vibrio cholerae $\mathrm{O} 1$ and a dfrA 15 cassette-containing integron in Vibrio parahaemolyticus isolated in Angola. Antimicrob Agents Chemother. 2006; 50(7): 2493-9. 